\title{
Mild Canavan disease
}

INSERM

\section{Source}

INSERM. (1999). Orphanet: an online rare disease and orphan drug data base. Mild Canavan disease. ORPHA:314918

Mild Canavan disease (CD) is a neurodeg enerative disorder characterized by mild speech delay or motor development. 